\section{Le vent du Jura souffle sur la Suisse}

La Société médicale du Canton du Jura, fondée en 1979 à la suite de l'entrée en souveraineté de la République et du Canton du Jura, a fêté son $20^{e}$ anniversaire. C'est donc avec quelque retard que nous dédions cette édition spéciale à la plus jeune société de médecine cantonale; mais qu'importe cette liberté prise avec le calendrier - une fois n'est pas coutume - puisqu'il s'agit avant tout de rendre hommage à nos collègues jurassiens qui ont accompli ces dernières années un travail d'organisation impressionnant, méritant à la fois la rétrospective historique et l'esquisse de perspectives futures. En leur donnant la parole, c'est aussi une façon de faire connaître une médecine pratiquée longtemps à l'écart des grands centres, assez méconnue et qui pourtant, par son originalité, offre un modèle dont on pourrait s'inspirer.

Une des caractéristiques les plus frappantes de la médecine dans le Jura est la rapidité avec laquelle elle est sortie d'une situation qu'on pouvait qualifier de moyenâgeuse pour se retrouver aujourd'hui à un niveau comparable - et compétitif - avec l'ensemble de la Suisse. Dans les entretiens avec nos collègues jurassiens, on ressent souvent la conscience vivante de cette évolution et de ces acquis médicaux qui ont eu leur prix et que l'on n'est pas prêt de sacrifier pour n'importe quoi; conscience qui va de pair avec une sensibilité politique et un goût de liberté que l'on connaît. La création du nouveau canton aura certainement eu l'avantage de pouvoir construire en même temps un système de santé taillé sur mesure - le rêve, probablement, de tout "health manager".

L'idée de cette édition spéciale est venue spontanément lors d'un entretien avec le Dr Pierre Philippe, président de la SMCJ. De fil en aiguille le projet s'est finalement réalisé et a mis au jour le dynamisme exceptionnel d'une société petite mais très vivante. Vous en trouverez le reflet dans ce journal qui représente un éventail de sujets les plus divers. J'en remercie vivement tous ceux qui y ont participé et tout particulièrement le Dr Philippe et son aimable épouse, Mme Carla Philippe, qui nous ont soutenu dans le travail rédactionnel avec précision et avec un grand soin des détails, ce qui aussi est typiquement jurassien.

Puisse ce souffle du Jura inciter d'autres sociétés de médecine cantonales, selon les occasions qui se présentent, à participer activement à notre travail; cela permettrait, de temps en temps, de descendre $d u$ haut des sphères politiques pour retrouver "dans le terrain" ce dont est fait le quotidien de la pratique médicale.

Markus Trutmann Rédacteur en chef

Conflit d'intérêt: l'auteur a des origines jurassiennes du côté maternel. 